\title{
DAS ANGST-MOTIV IN DER OCTAVIA DES PSEUDO-SENECA ${ }^{1}$
}

Key words: Octavia, pseudo-Senecan drama, passivity, resistance, Nero, Poppea.

In der Geschichte der römischen Literatur hat - wenn auch nicht wegen ihres literarischen Wertes, sondern wegen ihrer Kuriosität - die Octavia einen besonderen Platz, das Drama, das unter dem Namen des Denkers und Dichters Seneca überliefert worden ist und das die einzige fabula praetexta ist, die wir kennen. Diese Tragödie ist nicht leicht zu interpretieren, weil weder die Person ihres Autors noch ihre Verfassungszeit befriediegend geklärt sind. Die communis opinio ist - einerseits weil der Tod des Kaisers Nero von Agrippinas Geist so dargestellt wird, wie es bei den Geschichtsschreibern zu lesen ist, ${ }^{3}$ andererseits, weil es unwahrscheinlich wäre anzunehmen, daß der Autor seine Tragödie unter der neroischen Regierung veröffentlichen wollte -, daß dieses Werk nach dem Tod des Tyranns, also frühestens im Jahr $68 \mathrm{n}$. Chr. geschrieben wurde. ${ }^{4}$ Aber dieser terminus post quem läßt immer noch einen weiten Spielraum, um die Entstehungszeit der Octavia zu bestimmen. Selbst wenn man die Meinung, wonach diese Tragödie im 14. Jahrhundert entstand, unberücksichtigt läßt, bleibt immer noch ein 50jähriges Intervall (zwischen dem Tod des Nero und den Regierungsjahren von Traianus), in dem sie entstehen konnte. ${ }^{5}$ So schwer die genaue Entstehungszeit zu bestimmen ist, zumindest ebenso schwer ist der Ver-

\footnotetext{
${ }^{1}$ Dieser Vortrag wurde bei der Konferenz über das antike Drama in Pécs im Mai 2000 gehalten. ${ }^{2}$ Cf. SCHANZ-Hosius (1913), 2, pp. 73-76. Hosius, p. 75

Seneca: Tragedies. Ed. F. J. MILLER, Loeb Classical Library London pp. 460-461 und SCHANZ-

Cf. V. CIAFFI, Intorno all' autore dell' Octavia. RFIC 1937, pp. 246-265; M. E. CARBONE, The Octavia, Structure, date and authenticity. Phoenix XXXI (1977) pp. 48-67; Patrick Kragelund, The perfect's dilemma and the date of the Octavia. CQ XXXVIII (1988), pp. 492-508.

${ }^{5}$ SCHANZ-Hosius, p. 75.
} 
fasser der Tragödie zu identifizieren. ${ }^{6}$ Ich möchte hier nicht die Zahl der Vermutungen vermehren, sondern die Rolle des Motivs der Angst (timor, metus, pavor) in der Octavia, das sozusagen ein Leitmotiv der Tragödie zu sein scheint und mit dem sich die Fachliteratur nicht auseinandergesetzt hat, verfolgen und analysieren.

In diesem Drama erscheinen fünf historische Gestalten - deren Namen wir auch kennen - auf der Bühne: zuerst Octavia, die traurige Tochter des gestorbenen Kaisers Claudius und Messalina, die zur Zeit die gesetzliche Gemahlin Neros ist. Dann Seneca, der Erzieher des Kaisers; Nero, der legitime Kaiser, der sich von Octavia befreien will; und endlich Poppaea Sabina, die neue Begünstigte, die eine Ehe mit dem Tyrann schließen will. Die fünfte Figur ist die schon gestorbene Agrippina, deren Gespenst auftaucht, um die Umstände ihres Mordes in einem tragischen Mono$\log z u$ erläutern. Neben diesen fünf historischen Personen sehen wir einen unbenannten praefectus, der auch praefectus praetorio oder praefectus urbi, vielleicht praefectus vigilum sein kann, ${ }^{7}$ zwei Ammen (nutrix) und einen Boten (nuntius). Letztendlich gehören noch zwei Chöre zu den dramatischen Figuren. Die tragischen Ereignisse geschehen in den verschiedenen Teilen des kaiserlichen Palastes in Rom, im Jahre 62 n. Chr., Neros achtem Regierungsjahr. Eine Besonderheit des Werkes ist, daß die historischen Figuren einander nicht treffen, weswegen der dramatische Konflikt zwischen den Hauptfiguren auf der Bühne keinen Platz hat, weder Octavia und Nero, noch Octavia und Poppaea Sabina können vor dem Publikum aufeinanderstoßen. Die einzige Ausnahme ist Seneca, aber er - wie später gezeigt wird - spielt nur eine Nebenrolle, doch das Treffen mit Agrippina darf man außer acht lassen, weil nur ihr Gespenst erscheint, und auch nur für das Publikum sichtbar: in dem dramatischen Konflikt spielt die Mutter des Kaisers keine Rolle.

Wie oben angeführt, treten neben den historischen Gestalten zwei traditionelle dramatische Nebenfiguren auf, die Ammen, die für gewöhnlich die Rolle der Intriganten spielen. Die Hauptfiguren Octavia und Poppaea Sabina streiten sich mit ihnen, falls man überhaupt mit denen streiten kann, die einem zur Seite stehen. Den größten Teil des Dramas bilden die Zwiegespräche der Hauptfiguren und ihrer Vertrauten. Nach dem Monolog der Octavia, in dem sie die Umstände und ihre aussichtslose Situation darstellt, tritt ihre Amme auf, mit der die Kaiserin über die nächsten Schritte disputiert. Nach dem Auftreten der Chöre wird der Ort gewechselt, und wir sehen den Philosophen und Erzieher des Kaisers, Seneca, der darüber klagt, die Insel Corsica je verlassen zu haben, wo er ein den Denkern entsprechendes Leben hätte leben können. In diesem Augenblick tritt Nero auf, der inzwischen dem Präfekt einen Mordbefehl erteilt hat, und Nero erörtert mit Seneca die besten Regierungsformen. Es folgt der Monolog der Agrippina, dann Octavia und der mit ihnen sympathisierende Chor, und das Zwiegespäch der Poppaea Sabina, die ihre Heirat mit Nero vorbereitet, und ihrer Amme. In den letzten Szenen gibt es nur einen Dialog, das Zwiegespäch des wieder befehlenden Kaisers und des sich sträubenden Präfekten.

\footnotetext{
${ }^{6}$ Über die Autorschaft der Octavia: V. CIAFFI; Sullivan hat meiner Meinung nach recht: As for its authorship, only speculation is possible. In: J. P. SULLIVAN, Literature and Politics in the Age of Nero. Ithaca and London 1985 , p. 72

${ }^{7}$ Johanna SCHMIDT, Octavia. RE XVII, p. 1795.
} 
Es scheint, daß die Hauptfiguren weder miteinander noch mit denen, die zum anderen Kreis gehören, in Konfrontation geraten. Seneca ist keine Ausnahme, weil er nicht mit den Hauptfiguren, sondern mit den Ammen, den Vertrauten der neuen und der alten Frau auf gleicher Höhe steht. Das heißt, daß Octavia, Nero und Poppaea Sabina auf der obersten Stufe stehen und die zwei Ammen und Seneca (der in dem Drama kein unabhängiger Staatsmann, sondern der denkende Erzieher des Kaisers ist) eine unterworfene Rolle spielen. Auf der untersten Stufe stehen der Präfekt und der Bote, die bloße Befehlsvollzieher sind, obwohl der Präfekt versucht, den Kaiser eines Besseren zu belehren.

Wahrscheinlich läßt eine Tragödie, in der die Hauptfiguren den persönlichen Konflikt so sichtbar meiden, dem Tragikum wenige Möglichkeit, und es scheint, daß sich der einzige tragische Konflikt hinter den Kulissen abspielt: In der Octavia gibt es einen anderen, noch nicht benannten Beteiligten, den populus Romanus, das römische Volk. ${ }^{8}$ Die Ammen und Seneca berufen sich nämlich mehrmals auf den Volkswillen, auf die Popularität oder die Volksstimmung, die im Drama favor populi genannt wird. Das Publikum erfährt vom Boten, daß das römische Volk gegen die neue Ehe protestierend die Statuen der zukünftigen Frau Poppaea Sabina gestürzt hat. Das heißt, das Volk ist der einzige, der sich wagt, den Konflikt auf sich zu nehmen. Aber warum keine der anderen Figuren?

Es ist Morgen, gerade geht die Sonne auf, als Octavia eintritt und in Klagen ausbricht. Sie vergleicht sich mit Philomela und Elektra: sie hat niemanden, ${ }^{9}$ ihr Vater, ihre Mutter und ihr Bruder sind tot. In ihrem unermeßlichen Schmerz vereinigen sich Furcht und Angst. Aber die Angst, die in der Tragödie eine besondere Rolle spielt, ${ }^{10}$ kommt zuerst in den Worten der Amme zum Vorschein, nicht in ihrem Text. Ihre Amme ist ganz verzweifelt, sie sieht die neue Hochzeit, und sie kann keinen Rat geben, weil (was die Götter doch verhindern mögen!) ihre Furcht eine schreckliche Tat sieht: heu quam nefandum prospicit noster timor / scelus (vv. 55-56). ${ }^{11}$ Nicht ihr Vorgefühl, ihre Vorahnung, sondern ihre Angst vernimmt die Tat, die sich unaufhaltsam nähert.

Auch Octavia hat große Angst, aber nicht vor der Zukunft. Im Haus des Kaisers ist die Angst ihr Daseinselement. Im Gegensatz zu Elektra darf sie weder ihren Vater noch ihren Bruder betrauern: me crudeli sorte parentes/ raptos prohibet lugere timor / fratrisque necem deflere vetat ... (vv. 65-67). In dieser Hilflosigkeit und Einsamkeit will Octavia sterben, aber ihre Amme schlägt einen besseren Ausweg vor: Die Kaiserin muß versuchen, das Herz des Kaisers wiederzugewinnen. Octavia möchte zu keinen weiblichen Praktiken greifen, sie will nur sterben, aber sie hat auch vor dem Sterben Angst: trepidante semper corde non mortis metu / sed sceleris absit crimen a fatis meis, / mori iuvabit (vv. 106-108). Also nicht Todesangst hält sie zurück, obwohl ihr Herz erzittert, sondern die Angst vor der Schuld und das Verlan-

\footnotetext{
${ }^{8}$ SChanZ-HoSius, 74, Sullivan, 71-72.

${ }^{9}$ Peter L. ScHMidT, Die Poetisierung und Mythisierung der Geschichte in der Tragödie 'Octavia'. $A N R W$ II. 32.2 , p. 1426 .

${ }^{10}$ Zum Beispiel kommt das Wort timor, timeo dreizehnmal vor (vv. 55, 66, 111, 197, 211, 442, $457,734,736,755,800,858,871)$.

${ }^{11}$ Den Text zitiere ich aus der Loeb-Ausgabe.
} 
gen, daß ihr Schicksal (und ihr Name) rein bleibe. Aber was für eine Schuld ist es? Auf den ersten Blick scheint sie vor dem Selbstmord Angst zu haben, aber diese Lösung ist mit Senecas Ansicht über den Selbstmord oder der anderer zeitgenössischer Philosophien unmöglich in Beziehung zu setzen, weil eine wichtige Form des Protestes gegen Nero manchmal der freiwillige Tod war. (Es ist auszuschließen, daß man christliche Gedanken in diesem Werk findet.)

Antonio Santoro und die anderen Interpreten gehen von den Worten des Tacitus aus. Santoro meint, Octavia habe davor Angst, ein Opfer der Intrige des Kaisers zu werden, und er übersetzt den Ausdruck crimen als infamia. ${ }^{12}$ Diese Schande ist leicht mit dem in Beziehung zu bringen, was man in den Annales von Tacitus liest: Nero hat Octavia zweimal des Ehebruchs angeklagt, das erste Mal mit dem Flötenspieler Eucerus, dann mit Anicetus. ${ }^{13}$ Nach Santoro hat ihre Amme oder Sklavin die Kaiserin gerettet, und sie hat Angst davor, ein zweites Mal angeklagt zu werden.

Ohne Zweifel ist diese Interpretation nach Tacitus möglich, aber es findet sich in der Tragödie kein Wort davon, daß Octavia irgendwann einen Ehebruch begangen hätte. Damit ist auch schwer in Zusammenhang zu bringen, daß sie später sagt: poena nam gravior nece est / videre tumidos et truces miserae mihi / vultus tyranni, iungere atque hosti oscula / timere nutus (108-110). Demzufolge ist das Leben und die Tatsache, daß sie - wie früher - an der Seite des Tyrannen leben soll, eine größere Strafe für sie als der Tod. Aber meiner Meinung nach muß dieses Zitat im Licht des Vorigen interpretiert werden. Wenn man bei dieser Interpretation dem Historiker Tacitus folgt, dann ist es logisch, daß Nero wegen des Ehebruches (crimen) seine Frau Octavia verstößt. In diesem Fall aber würde Octavia nicht dazu gezwungen, den verhaßten Mann immer anzusehen und Angst zu habe. Die rechte Interpretation der Worte der Octavia findet sich in den Worten ihrer Amme. Was sie scelus nennt, das ist auch das scelus für Octavia.

Die Handlung der Tragödie spielt vor der Ehe Kaiser Neros mit Poppaea Sabina, der neuen Frau, und alle Ereignisse hängen damit zusammen. Was die Amme früher scelus nannte, bezieht sich auch auf diese neue Heirat. Das heißt, Octavia, Neros legitime Frau, weist den Selbstmord zurück, weil sie die Lage des Kaisers nicht erleichtern, sich nicht freiwillig aus dem Wege ihres Mannes stehlen will. Es ist ein Paradox, daß die dramatische Tat der Octavia sich nicht im Handeln, sondern in ihrer Untätigkeit zeigt. Sie ist eine angstvolle und gleichzeitig beständig ausdauernde Heldin. Sie fürchtet ihren Mann, den Kaiser, wie einen Gott, sie hat Angst vor seinem Kopfnicken: timere nutus.

In dieser verzweifelten Situation bittet Octavia um die Hilfe ihres Vaters, aber die Worte ihrer Amme ernüchtern sie: Ihr Vater wird nicht zur Hilfe eilen, denn er hat zugelassen, daß ein adoptierter Flegel die Stelle seines Sohnes einnehme. Aus den Worten der Amme geht hervor, daß Octavias Bild von ihrem Vater Claudius falsch ist. Er ist in großem Maße verantwortlich dafür, daß seine Tochter so schreckliche Dinge ertragen muß, weil er Agrippina zur Frau genommen hat: hic orta series

\footnotetext{
${ }^{12}$ Incerti poetae Octavia. Testo introduzione e commento a cura di Antonio SANTORO. Bologna 1955, p. 31. n. ad vv. 107-109.

${ }^{13} \mathrm{Cf}$. Tacitus, Annales XIV, 60; 62 .
} 
facinorum (143). Es war die Intrige dieser Stiefmutter, oder wie die Amme sie nennt, dira genetrix, daß Octavia die Frau des Nero wurde, Agrippina et te iunxit invitam metu (154). Bei diesem Ausdruck ist nicht zu entscheiden, auf welches Wort im Satz das Wort in Ablativ, metu, sich bezieht. Man weiß, daß Octavia im Jahre 39/40 geboren, und am 29. August $41 \mathrm{n}$. Chr. noch als Säugling mit Iunius Silanus, der in der Tragödie auch erwähnt wird, verlobt wurde. Sieben Jahre später wurde diese Verlobung aufgelöst, und das ungefähr zehnjährige Mädchen wurde die Verlobte Neros. Octavia war erst vierzehn Jahre alt, als - ein Jahr vor dem Tod des Claudius die Hochzeit stattfand. Octavia muß also den jungen Ahenobarbus sehr gut gekannt haben, weil sie mehrere Jahre lang in dem Palast nebeneinander gelebt hatten, obwohl es wirklich zweifelhaft ist, ob eine Zehnjährige ihrem Verlobten treu bleiben konnte. Jedenfalls würde ihre frühere Antipathie gegen Nero erklären, daß sie ihm auch später abgeneigt war.

Aber die grammatische Konstruktion schließt nicht aus, daß sich das Wort metu auf Agrippina bezieht: Agrippina wurde von der Angst gezwungen, ihren Sohn zum Mann der Tochter des Claudius zu machen, um Nero den Thron zu sichern. Aus den historischen Quellen geht hervor, wie entschlossen Agrippina dafür gekämpft hat, daß ihr Sohn Kaiser werde, und man weiß auch, daß sie statt ihres Sohnes - der ja noch ein Kind war - selbst das Reich regieren wollte. So ist die Angst im Falle Agrippina gerechtfertigt, denn sie fürchtete, daß Britannicus ihrem Sohn zuvorkommt.

Im Dialog der Octavia und ihrer Amme kommt man auf die Ermordung des Kaisers zu sprechen, obwohl Octavia eher wünscht, von Nero umgebracht zu werden als umgekehrt. Auch die Amme zweifelt daran, daß in der Kaiserin solche Kraft whone, aber: dolor, ira, maeror, miserae, luctus dabunt (176). Deshalb schlägt sie vor, Octavia solle das Herz Neros wiedergewinnen: vince obsequendo potius immitem virum (177). Und falls Octavia nicht genug Kraft hätte - und hier tritt das bis zuletzt hinter den Kulissen verborgene Volk in die Handlung ein -, werden die Römer ihr diese Kraft geben. Der Meinung der Amme nach hat der populus Romanus die gröBere Kraft, doch Octavia meint, der princeps sei doch stärker. Octavia verrät, daß ihre Angst nicht nur Nero gilt, sondern auch Poppaea Sabina, weil der Kaiser in sie verliebt ist und Poppaea ihn fest in der Hand hat. Die Amme sagt, daß die Liebe des Kaisers vergänglich sei, und nennt als Beispiel die Geschichte der griechischen Sklavin, die auch Neros Herz eroberte, ihm später aber lästig wurde; letztendlich blieb ihr nur noch die Furcht: animumque domini famula possedit diu, / iam metuit eadem (194-195).

Als der Chor der mit Octavia sympathisierenden Römerinnen endlich die Einsamkeit der Kaiserin begreift, macht er sich selbst Vorwürfe: nos quoque nostri sumus immemores / post fata ducis, cuius stirpem / prodimus aegro suadente metu (288-290). Das heißt, sie haben Angst, ihre Sympathie für Octavia auszudrücken, obwohl dies ihre persönliche Heldentat bedeuten würde.

Am Anfang des zweiten Aktes steht Seneca auf der Bühne und trägt dem Publikum seinen Monolog vor, in dem er sich beklagt, warum er nicht auf Korsika geblieben ist, um das Leben eines Philosophen zu führen: melius latebam (381), aber 
sein Schicksal hat ihn in die Höhe gehoben, daß gravius ut ruerem edita / receptus arce totque prospicerem metus? (379-380). Man erinnert sich an Lucretius, was der Weise begehrt. Aber Seneca betrachtet die Welt nicht mit Ruhe: was er sieht, ist eine Vielfalt der Angst, die die Welt beherrscht. Und Seneca selbst ist auch keine Ausnahme, weil er am Ende seines Monologs, der sich auf die Metamorphoses von Ovid beruft, Nero anblickend folgendes sagt: quid ferat mente, horreo (437).

Und Nero, als ob er sofort die ihn umgebende Furcht beweisen wollte, gibt dem begleitenden Präfekten Befehle, wer umgebracht werden soll. Die Todesbefehle geben Seneca eine gute Gelegenheit darzulegen, was für ihn ein guter Herrscher bedeutet. Sie verwickeln sich in einen Disput. Aber Nero verrät, daß er auch selbst Angst hat: iustum esse facile est cui vacat pectus metu (441). Seine Worte zeigen, $\mathrm{da}$ er in seiner Politik von der Angst geleitet wird: er klammert sich an die Macht und muß seine Gegner beseitigen, so daß sie keine Bedrohung für sein Primat darstellen. ${ }^{14}$

Der Streit, dessen Fundament zweifellos der Dialog von Seneca De clementia ist, wird nicht ausführlich dargelegt. Der Höhepunkt dieses Streites ist die Interpretation der augustischen Herrschaft. Seneca sagt, die Tugend der clementia habe nach dem Bürgerkrieg die Regierung von Augustus gekennzeichnet, Neros Meinung nach war jedoch die Abrechnung mit seinen Gegnern immer ein wichtiges Element seiner Machtversicherung: et continuit imperium metus (526).

Aber dieses Zwiegespräch handelt nicht nur von der Macht; Seneca weist auch darauf hin, daß Octavia ein Kind für Nero zur Welt bringen wird und diese Lösung für das Volk beruhigend und die einzig akzeptable Alternative wäre. Das beweist auch, daß sich die in der Tragödie mitgeteilten Gedanken und Ereignisse - wenn oft auch nicht direkt - auf die vorbereitende ungesetzliche Ehe beziehen.

In der dritten Szene bittet Octavia das Volk, nicht seine Sympathie auszudrükken, weil es sich dann selbst ins Unglück stürzt, und der Chor selbst formuliert es auch, daß es Octavia die pietas wegen seiner Angst verweigert.

Aber nicht nur Octavia und das römische Volk haben Angst, sondern auch die in der vierten Szene auftretende Poppaea Sabina, die ihre tiefsten Gedanken ihrer Amme mitteilt. Poppaea hat etwas Schreckliches geträumt und kann diesen Alpdruck nicht loswerden: confusa tristi proximae noctis metu / visuque, nutrix, mente turbata feror / defecta sensu ... (712-714). In ihrem Traum sind Agrippina und die römischen Frauen vorgekommen, ihr Mann ist auch erschienen, und als er Poppaea küssen wollte, ist Nero ins Zimmer hereingestürmt, um den Mann zu erdolchen. Die Angst (oder das schlechte Gewissen) hat sie geweckt. Ihre Amme beruhigt sie; dann tritt der Bote ein und berichtet Poppaea, daß das Volk sich aufgelehnt hat und, einmalig in der Tragödie, victa nec cedit metu / concepta rabies temere, sed vires capit (783-784). Der einzige, der in der Octavia seine Angst loswird, ist das römische Volk, der populus Romanus, das nicht auf Octavias Warnungen hört und sich gegen den Kaiser stellt. Diese Tat des Volkes ist jedoch zum Scheitern verurteilt und löst nur den Zorn Neros aus, der seinem Präfekten befiehlt, Octavia zu ermorden. Im letz-

${ }^{14}$ Cf. Seneca, Agamemnon vv. 71-76. 
ten Bild des Dramas fährt das Schiff mit der verbannten Octavia nach Pandeteria (wo sie später auch gestorben ist).

Die meisten sehen den einzigen tragischen Akt des Dramas in dem Aufstand des römischen Volkes. ${ }^{15}$ Wie es gezeigt wurde, sind alle Gestalten Gefangene ihrer Ängste: Poppaea tut aus Angst nichts, Seneca hält sich aus den Ereignissen heraus, der Präfekt hat auch Angst, sie bringt ihn dazu, die Befehle des Herrschers zu erfüllen. Es ist eine andere Form der Angst, aber auch der Kaiser fürchtet sich. Eine spezielle, mit den anderen Ängsten nicht verwandte Furcht knebelt Octavia, deren Fall nur teilweise durch den Aufstand des Volkes verursacht wird. Die Ursache ihres tragischen Sturzes ist ihre Standhaftigkeit.

So ist meiner Meinung nach auch Octavia eindeutig eine tragische Gestalt, aber ihre Tat, die man von einer dramatischen Heldin erwartet, besteht paradoxerweise nicht in der Aktivität, sondern in ihrer Passivität, in ihrer Standhaftigkeit. Für sie bedeutet die neue Ehe den Tod und die endgültige Liquidierung des claudischen Erbes. Die Octavia ist eine Tragödie, die Tragödie der Angst und der aktiven Passivität.

Pázmány Péter Catholic University Department of Classical Philology H-2087 Piliscsaba, Egyetem u. 1.

${ }^{15}$ Sullivan, 70. 